\title{
Abnormal thyroid hormone response to TRH in a case of macro-TSH and the cut-off value for screening cases of inappropriate TSH elevation
}

\author{
Kenji Ohba ${ }^{1)}$, Masato Maekawa ${ }^{2)}$, Kunihiro Iwahara' ${ }^{2)}$, Yasuhide Suzuki ${ }^{3)}$, Akio Matsushita ${ }^{1)}$, \\ Shigekazu Sasaki ${ }^{1)}$, Yutaka Oki ${ }^{1,4)}$ and Hirotoshi Nakamura ${ }^{1,5)}$ \\ 1) Second Division, Department of Internal Medicine, Hamamatsu University School of Medicine, Hamamatsu, Shizuoka 431-3192, Japan \\ 2) Department of Laboratory Medicine, Hamamatsu University School of Medicine, Hamamatsu, Shizuoka 431-3192, Japan \\ 3) Department of Laboratory Medicine, Enshu Hospital, Hamamatsu, Shizuoka 430-0929, Japan \\ 4) Department of Family and Community Medicine, Hamamatsu University School of Medicine, Hamamatsu, Shizuoka 431-3192, Japan \\ 5) Department of Internal Medicine, Kuma Hospital, Kobe, Hyogo 650-0011, Japan
}

\begin{abstract}
A 74-year-old asymptomatic Japanese man with suspected thyroid dysfunction was referred to our hospital. He had an elevated TSH (53.8 mIU/L; reference interval: 0.5-5.0) despite a free T4 (FT4) level (1.4 ng/dL; reference interval: 0.91.6). Further analysis revealed macro-TSH. A notable finding was that a $500-\mu \mathrm{g}$ TRH stimulation test revealed a blunted free T3 (FT3) response despite a prolonged TSH response. Macro-TSH typically presents with inappropriately marked elevation of serum TSH levels compared with other thyroid hormones, as exhibited in our case. However, the level of TSH elevation that might differentiate macro-TSH from subclinical hypothyroidism is poorly known. We retrospectively analyzed 8,183 concurrent measurements of TSH and FT4 in individuals previously examined in our hospital to define the cut-off value for screening cases of inappropriate TSH elevation. FT4 values were rounded off to one decimal place, and the 97.5th percentile of TSH against each FT4 value was calculated. The data of our patient and that of 30 cases of macro-TSH extracted from the English literature were then assessed. When the approximate curve obtained from the 97.5th percentile of TSH values was defined as the cut-off value $\left[\log _{10} \mathrm{TSH}=0.700+1.549 /\left\{1+(\mathrm{FT} 4 / 0.844)^{6.854}\right\}\right], 25$ of the $31(80.6 \%)$ macro-TSH cases were identified. In conclusion, we report for the first time a case of macro-TSH demonstrating an abnormal FT3 response to TRH. A cut-off value of TSH adjusted to the FT4 level may be a good method of screening for inappropriate TSH elevation (or inappropriate hyperthyrotropinemia) including those caused by macro-TSH.
\end{abstract}

Key words: Macro-thyrotropin, Inappropriate TSH elevation, TRH stimulation test, Thyroxine, Triiodothyronine

MACRO-THYROTROPIN (MACRO-TSH) is a high molecular weight form of TSH that typically results in inappropriate elevation in serum TSH concentrations despite thyroid hormone concentrations within the reference intervals $[1,2]$. The major cause is thought to be anti-TSH antibodies, although the pathogenesis is not fully clarified [3-5]. The TSH-antibody complexes are not efficiently excreted by the kidneys because of their large molecular size, resulting in accumulation in the serum. The antibody-bound TSH is immunoreactive but hormonally inactive, leading to isolated elevation in serum TSH concentration. Macro-TSH is primarily

Submitted Jul. 22, 2019; Accepted Sep. 23, 2019 as EJ19-0320 Released online in J-STAGE as advance publication Oct. 24, 2019 Correspondence to: Kenji Ohba, MD, PhD, Medical Education Center, Hamamatsu University School of Medicine, 1-20-1 Handayama, Higashi-ku, Hamamatsu, Shizuoka 431-3192, Japan.

E-mail: ohbak@hama-med.ac.jp regarded as a type of assay interference, although its possible effect on thyroid hormone homeostasis remains controversial $[2,5]$.

Although macro-TSH typically presents with marked elevation of serum TSH levels relative to other thyroid hormones [6-8], similar patterns of abnormal hormone levels have also been reported in patients with resistance to thyroid hormone $\beta$ coexisting with Hashimoto's thyroiditis [9] or in those being treated with tyrosine kinase inhibitors [10]. However, little is known about the level of TSH elevation that might differentiate macro-TSH from subclinical hypothyroidism [2, 11]. Because small changes in circulating free T4 (FT4) concentrations are known to result in relatively large changes in TSH, we previously analyzed the TSH-FT4 relationship and proposed reference intervals of TSH according to different FT4 levels [12].

In the present study, we report a unique case of macro- 
TSH in which there was an abnormal free T3 (FT3) response to a TRH stimulation test. Based on data from the literature, we also evaluated a cut-off value to screen cases of inappropriate TSH elevation, including those caused by macro-TSH.

\section{Patient}

A 74-year-old asymptomatic Japanese man with chronic hepatitis $\mathrm{C}$ was referred to Hamamatsu University School of Medicine because of suspected thyroid dysfunction noted on a screening blood test performed prior to interferon therapy. On physical examination, the patient was clinically euthyroid. Routine blood chemistries showed mild anemia and slightly elevated hepatic transaminase levels. Thyroid function tests (TFTs) showed a markedly elevated TSH $(53.8 \mathrm{mIU} / \mathrm{L}$; reference interval: $0.5-5.0)$ despite having the levels of FT4 $(1.4 \mathrm{ng} / \mathrm{dL}$; reference interval: $0.9-1.6)$ and FT3 (2.7 $\mathrm{pg} / \mathrm{mL}$; reference interval: $2.3-4.0$ ) measured with the Elecsys platform (Roche Diagnostics). Repeat measurement using another analytical platform (ADVIA Centaur, Siemens) also revealed a high TSH level $(66.6 \mathrm{mIU} / \mathrm{L}$; reference interval: 0.4-4.0). We conducted the following examinations.

\section{Methods}

A polyethylene glycol (PEG) precipitation test, gel filtration chromatography, and TRH stimulation test were performed as described elsewhere [13]. Two hours after TRH administration, total serum T3 and/or free T3 concentrations in healthy volunteers reportedly increase by approximately $30 \%[14,15]$. PEG precipitation after $\mathrm{HCl}$ incubation was conducted, with some modifications, according to a previous study using this test to identify macroprolactinemia [16]. Because an acidified buffer dissociates the antigen-antibody complex, this method theoretically precipitates only antibodies. Written informed consent was obtained from the patient for the publication of this case report, and the institutional review board of the Hamamatsu University School of Medicine approved its publication.

To determine a cut-off value of TSH levels with respect to different FT4 levels, we retrospectively reviewed concurrent TFT measurements from 8,183 individuals seen at Enshu Hospital for the first time between July 2008 and June 2016 (Fig. 1A). TSH and FT4 concentrations were determined using the Elecsys platform (Roche Diagnostics). Reference intervals of these hormones in Enshu Hospital were 0.5-5.0 mIU/L and $0.9-1.7 \mathrm{ng} / \mathrm{dL}$, respectively. Patients with any type thyroid dysfunction were included, irrespective of

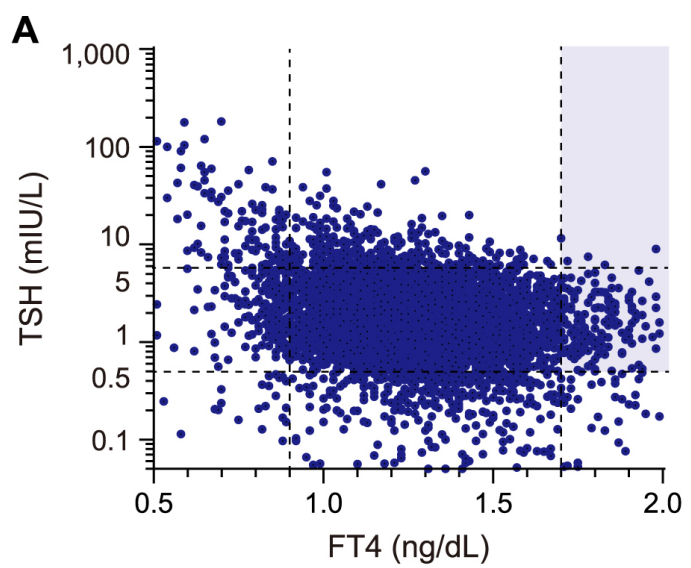

B
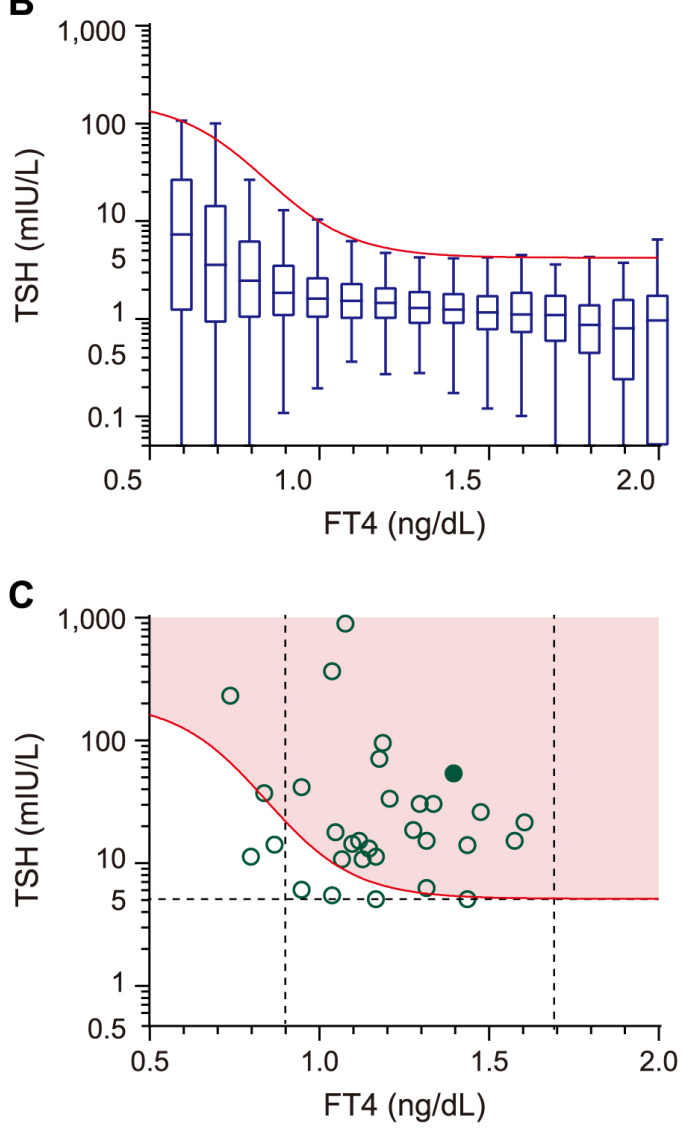

Fig. 1 Cut-off value of thyroid function test for screening cases with inappropriate TSH elevation (or inappropriate hyperthyrotropinemia), including those caused by macro-TSH. (A) Distribution of TSH and free T4 (FT4) in 8,183 individuals. Dotted horizontal and vertical lines mark the reference intervals of TSH $(0.5-5.0$ $\mathrm{mIU} / \mathrm{L})$ and FT4 (0.9-1.7 ng/dL), respectively. The shaded region represents the area of syndrome of inappropriate secretion of TSH (SITSH), or central hyperthyroidism [28]. (B) Box and whisker plots denote the 2.5 th, 25 th, 50th (median value), 75 th, and 97.5 th percentiles of the TSH values against each FT4 value (from 0.5 to $2.0 \mathrm{ng} / \mathrm{dL}$ ) rounded to one decimal place. The approximate curve (solid curve) is derived from the cut-off value obtained from the 97.5th percentile of each TSH value using a four-parameter logistic model $\left[\log _{10} \mathrm{TSH}=0.700+1.549 /\left\{1+(\mathrm{FT} 4 / 0.844)^{6.854}\right\}\right]$. (C) Distribution of TSH and FT4 in the present case (filled circle) and 30 reported cases (open circle) of macro-TSH [5-8, 11, 20-24]. The area above the cut-off value (shaded area) indicates that most macro-TSH cases were correctly identified by this proposed definition of the cut-off value. 
whether they were receiving drug therapy. The institutional review board of the Enshu Hospital approved the study protocol and waived the need for written informed consent because of its retrospective nature.

FT4 levels were rounded to one decimal place and the 2.5th and 97.5th percentiles of the TSH levels were calculated against each FT4 value (Fig. 1B). The reason for this method is that it is reported that the TSH-FT4 relationship is not inverse log-linear but rather resembles negative sigmoid curves [17]. Approximate curve was derived from 97.5th percentiles of each TSH value using a four-parameter logistic model. The TSH-FT4 relationship was calculated as follows: $\log _{10} \mathrm{TSH}=0.700$ $+1.549 /\left[1+(\mathrm{FT} 4 / 0.844)^{6.854}\right]$. The statistical analyses were performed using GraphPad PRISM v7.0 (GraphPad Software, Inc.).

We conducted a MEDLINE search using the keyword "macro-TSH" or "macro-thyrotropin" in articles published in English between 1998 and 2018. Reports were excluded if they did not include individual values and/or reference intervals for TFTs. FT4 and TSH values in each case reported were adjusted by the upper reference limit in each institution, according to a method (with some modifications) used in a study summarizing the distribution of TSH and FT4 in patients with resistance to thyroid hormone $\beta$ [18]. The adjusted TSH value was calculated as follows: reported TSH value $\times 5.0$ (upper TSH reference limit in Enshu Hospital)/upper TSH reference limit in the published case.

\section{Results}

PEG precipitation of TSH demonstrated an extremely low recovery rate $(2.8 \%)$ (Table 1$)$. After the serum was acidified with $\mathrm{HCl}$, however, the recovery rate was high, suggesting the presence of a high serum TSH concentration. TSH is a heterodimer of $\alpha$ ( $\alpha$ subunit of glycoprotein hormone, $\alpha \mathrm{GSU}$ ) and $\beta$ (TSH $\beta$ ) chains, and $\alpha \mathrm{GSU}$ is common to LH and FSH. Because the recovery rate of these hormones was not significantly reduced, the assay interference in this case was considered to be specific to
TSH $\beta$. An aliquot of the serum sample was sent to the laboratory company, where further analysis was conducted as previously reported [19]. Briefly, human antimurine antibody (HAMA) interference, a common heterophilic interference, was excluded by using a specific TSH reagent. The patient's serum was further fractionated by gel filtration chromatography of $\mathrm{pH} 7.2$ eluent and TSH was measured in each fraction (Fig. 2). The presence of TSH was illustrated by one peak located at a position consistent with a slightly larger molecular weight than the IgG peak. Elution patterns of FT4 and FT3 (pH 7.2) were coincident with those in the control subject. Subsequent gel filtration chromatography was performed with $\mathrm{pH} 3.0$ eluent to dissociate the antigenantibody complex. The elution time of the TSH peak changed from $28.0 \mathrm{~min}$ to $33.0 \mathrm{~min}$, demonstrating an elution pattern similar to that previously reported in macro-TSH [6].

Based on these findings, the patient was diagnosed with macro-TSH. Interestingly, a TRH stimulation test

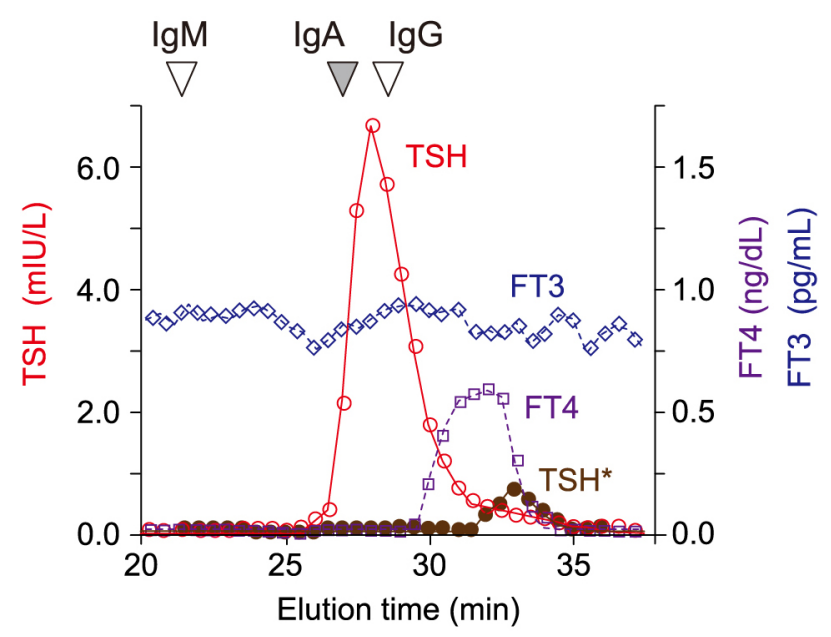

Fig. 2 Gel filtration chromatography. The peak fractions of IgM, $\mathrm{IgA}$, and $\mathrm{IgG}$ are shown as markers of molecular mass. The open circle, square, and diamond indicate TSH, free T4 (FT4), and free (FT3), measured with pH 7.2 eluent. *TSH was measured with pH 3.0 eluent (solid circle).

Table 1 Polyethylene glycol precipitation test

\begin{tabular}{lccc}
\hline & Before PEG Treatment & After PEG treatment & Recovery rate (\%) \\
\hline TSH $(\mathrm{mIU} / \mathrm{L})$ & 53.8 & 1.5 & $2.8[60.4 \pm 14.6]$ \\
TSH* $(\mathrm{mIU} / \mathrm{L})$ & 53.8 & 44.1 & $82.0[77.7 \pm 20.3]$ \\
$\mathrm{LH}(\mathrm{mIU} / \mathrm{mL})$ & 6.4 & 2.9 & $45.4[44.7 \pm 5.0]$ \\
FSH $(\mathrm{mIU} / \mathrm{mL})$ & 9.5 & 9.6 & $101.6[106.3 \pm 7.9]$ \\
\hline
\end{tabular}

The recovery rate was calculated as recovery $(\%)=$ value after polyethylene $(\mathrm{PEG})$ treatment/value before PEG treatment $\times 100$. ${ }^{*} \mathrm{TSH}$ obtained after serum incubated with $\mathrm{HCl}$ and then subjected to PEG. Data in square brackets are mean $\pm 2 \mathrm{SD}$ in control serum $(n=8)$. 


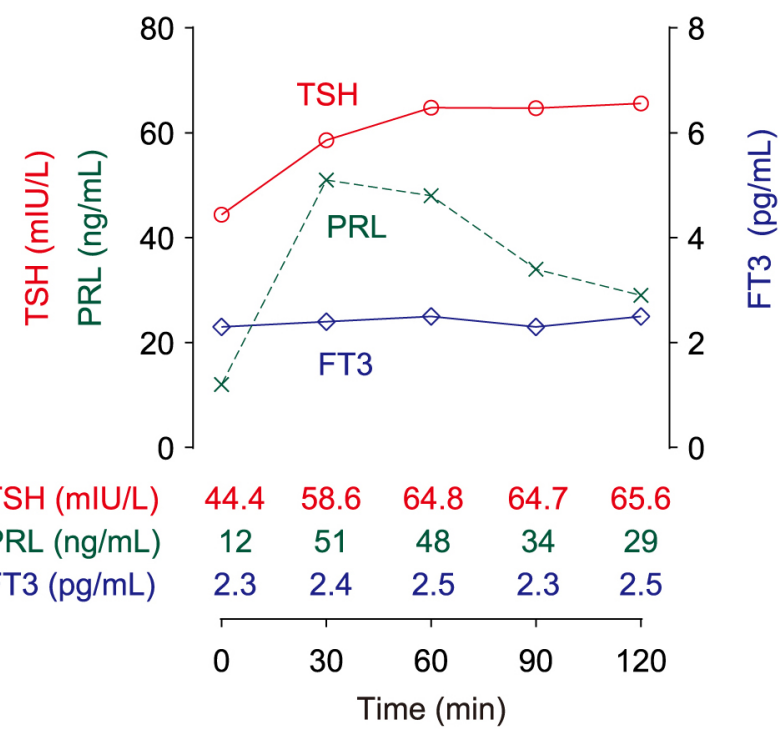

Fig. 3 Prolonged TSH and blunted free T3 (FT3) responses to TRH stimulation. Serum hormones were measured at indicated time points after injection of $500 \mu \mathrm{g}$ of TRH.

revealed a prolonged TSH response but a blunted FT3 response despite a normal PRL response (Fig. 3). No pituitary adenoma was revealed in contrast-enhanced computed tomography. During 24 months of follow-up, the patient developed no thyrotoxic signs or symptoms. Results of periodic measurements of TFTs remained stable, with continued marked elevation of the TSH level but thyroid hormone levels within the reference intervals.

The approximate curve obtained from 97.5th percentiles of TSH values was defined the cut-off value to screen the cases of inappropriate TSH elevation, including those caused by macro-TSH (Fig. 1C). The literature search yielded 30 adult cases of macro-TSH in 10 references [5-8, 11, 20-24]. Including the values from our patient, the TSH levels were higher than the cut-off values for the cases of inappropriate TSH elevation in 25 of the $31(80.6 \%)$ cases.

\section{Discussion}

In addition to macro-TSH, other conditions that may interfere with routine TFT assays include heterophilic antibodies, thyroid hormone autoantibodies, and assayspecific interference [2]. The mechanism of interference with thyroid function immunoassays is classified as cross-linking or autoimmune complexes against hormones. Heterophilic antibodies including HAMA are involved in the cross-linking type, where capture- and detection-antibodies are bridged despite the absence of $\mathrm{TSH}$, resulting in falsely high TSH values reported in a sandwich-type immunoassay system. In contrast, macro-
TSH belongs to the latter type of interference. As shown by the results of PEG precipitation after incubation with $\mathrm{HCl}$ (Table 1), the serum concentration of TSH indeed was high, but it was present as both the free and immunoglobulin-bound forms. Although several studies have reported that macro-TSH results in falsely, spuriously, or factitiously high TSH despite normal thyroid hormone levels $[2,7,8,20]$, the terms "false, spurious, or factitious" are better used in interference due to crosslinking rather than in macro-TSH.

Several researchers have conducted TRH stimulation tests in individuals with macro-TSH; however, neither free nor total thyroid hormone levels were reported in their studies $[1,25,26]$. In the present case, a TRH stimulation test revealed a blunted FT3 response in addition to a prolonged TSH response (Fig. 3). To the best of our knowledge, this is the first report of this phenomenon. As a possible mechanism, most free (immunoglobulinunbound) TSH secreted after TRH stimulation was likely rapidly bound to antibodies, forming the hormonally inactive immunoglobulin-bound macro-TSH, which therefore did not increase serum FT3. Although only one case report, our findings suggest that macro-TSH may interfere with the acute response of circulating T3 levels to TRH stimulation. Hattori et al. recently reported two patients whose elevated free TSH levels returned to normal after macro-TSH spontaneously resolved [5]. The authors also proposed that macro-TSH is a possible cause of hyperthyrotropinemia. Based on these findings, it is possible that macro-TSH may not represent merely in vitro assay interference. Further studies are needed to evaluate whether macro-TSH could exert an effect on thyroid hormone homeostasis, including the hypothalamus-pituitary-thyroid axis (e.g., the response of circulating TSH and thyroid hormone concentrations to TRH stimulation).

Subclinical hypothyroidism is defined a serum TSH concentration above the upper reference limit despite thyroid hormone levels within the reference range. However, there is little information about the level of TSH elevation that may differentiate macro-TSH from subclinical hypothyroidism. Milles et al. reported that macro-TSH should be suspected in a patient with a TSH concentration $>10 \mathrm{mIU} / \mathrm{L}$ when thyroid hormone levels are within reference intervals [11]. Other groups proposed that isolated TSH elevation with thyroid hormones in the upper half of the reference intervals indicate possible macro-TSH [2]. Because circulating thyroid hormones are the most significant factor contributing to serum TSH levels, we defined the cut-off value for screening for macro-TSH in terms of individual FT4 levels. Subsequent literature review demonstrated that TFTs obtained from $80.6 \%$ (25/31) reported cases of macro- 
TSH were plotted in our proposed range. Because of the relatively rare prevalence of macro-TSH, ranging from $0.17 \%$ to $1.6 \%[11,22-24]$, it is difficult to establish a firm cut-off to exclude macro-TSH in all patients who in fact have subclinical hypothyroidism. Our proposed method using different cut-off intervals of TSH according to the patient's FT4 level can make the differential diagnosis of subclinical hypothyroidism easier. Further studies evaluating both sensitivity and specificity are needed to validate the cut-off value for inappropriate TSH elevation.

The diagnostic strategy for investigating macro-TSH after screening remains debated, given that no single method can reliably diagnose macro-TSH at present. Even gel filtration chromatography, which is the preferred method for identifying macro-TSH, is not sensitive in cases wherein TSH and the antibodies are weakly bound [24]. Hence, the physician should perform several methods, including PEG method and gel filtration chromatography, which are expensive and/or complicated to perform. In addition to a cut-off definition of TSH levels with respect to different FT4 levels, a TRH stimulation test is simple and relatively inexpensive. Combining these methods could contribute to the differential diagnosis in patients with isolated TSH elevation, including those caused by macro-TSH. Further studies are needed to evaluate their diagnostic usefulness.

As a limitation of our study, patients with any type of thyroid disorders were included to determine the cut-off value for inappropriate TSH elevation. The TSH-FT4 relationship is influenced by several factors including age, sex, medication, and the history of thyroidectomy. It has been also reported that serum TSH can be drastically suppressed, sometimes for weeks, following the recovery of thyrotoxicosis [27]. Further studies are required to validate the cut-off value after considering the confounders of TSH-FT4 relationship.

In conclusion, we report for the first time an abnormal thyroid hormone response against TRH stimulation in a case of macro-TSH. Our calculations using data from the literature of patients with macro-TSH suggests that a cutoff value of TSH based on individual FT4 levels may be a useful method of screening for cases demonstrating inappropriate TSH elevation (or inappropriate hyperthyrotropinemia), including those caused by macro-TSH.

\section{Acknowledgements}

The authors are grateful to all the doctors who provided us with the opportunity to study the clinical course of this patient. We also thank Enago (www.enago.jp) for the English language review.

\section{Disclosure}

None of the authors have any potential conflicts of interest associated with this research.

\section{References}

1. Spitz IM, Le Roith D, Hirsch H, Carayon P, Pekonen F, et al. (1981) Increased high-molecular-weight thyrotropin with impaired biologic activity in a euthyroid man. $N$ Engl J Med 304: 278-282.

2. Favresse J, Burlacu MC, Maiter D, Gruson D (2018) Interferences with thyroid function immunoassays: clinical implications and detection algorithm. Endocr Rev 39: 830-850.

3. Akamizu $\mathrm{T}$, Ishii $\mathrm{H}$, Mori $\mathrm{T}$, Ishihara $\mathrm{T}$, Ikekubo $\mathrm{K}$, et al. (1984) Abnormal thyrotropin-binding immunoglobulins in two patients with Graves' disease. J Clin Endocrinol Metab 59: 240-245.

4. Ikegami K, Liao XH, Hoshino Y, Ono H, Ota W, et al. (2014) Tissue-specific posttranslational modification allows functional targeting of thyrotropin. Cell Rep 9: 801-810.

5. Hattori N, Ishihara T, Matsuoka N, Saito T, Shimatsu A (2017) Anti-thyrotropin autoantibodies in patients with macro-thyrotropin and long-term changes in macrothyrotropin and serum thyrotropin levels. Thyroid 27: 138-146.

6. Sakai H, Fukuda G, Suzuki N, Watanabe C, Odawara M
(2009) Falsely elevated thyroid-stimulating hormone (TSH) level due to macro-TSH. Endocr J 56: 435-440.

7. Verhoye E, Van den Bruel A, Delanghe JR, Debruyne E, Langlois MR (2009) Spuriously high thyrotropin values due to anti-thyrotropin antibodies in adult patients. Clin Chem Lab Med 47: 604-606.

8. Loh TP, Kao SL, Halsall DJ, Toh SA, Chan E, et al. (2012) Macro-thyrotropin: a case report and review of literature. J Clin Endocrinol Metab 97: 1823-1828.

9. Fukata S, Brent GA, Sugawara M (2005) Resistance to thyroid hormone in Hashimoto's thyroiditis. $N$ Engl J Med 352: $517-518$.

10. Ohba K, Takayama T, Matsunaga H, Matsushita A, Sasaki $\mathrm{S}$, et al. (2013) Inappropriate elevation of serum thyrotropin levels in patients treated with axitinib. Thyroid 23: 443-448.

11. Mills F, Jeffery J, Mackenzie P, Cranfield A, Ayling RM (2013) An immunoglobulin G complexed form of thyroidstimulating hormone (macro thyroid-stimulating hormone) is a cause of elevated serum thyroid-stimulating hormone concentration. Ann Clin Biochem 50: 416-420.

12. Ohba K, Sasaki S, Nakamura H (2014) Proposal for the 
tentative range of inappropriate TSH elevation. Program of 84th Scientific Sessions of American Thyroid Association, A96 (Abstract).

13. Ohba K, Shirakawa K, Okawa Y, Iwaki H, Matsunaga H, et al. (2011) Syndrome of inappropriate secretion of thyrotropin associated with thymoma-related peripheral nerve hyperexcitability. Endocr J 58: 597-602.

14. Butler PW, Smith SM, Linderman JD, Brychta RJ, Alberobello AT, et al. (2010) The Thr92Ala 5' type 2 deiodinase gene polymorphism is associated with a delayed triiodothyronine secretion in response to the thyrotropinreleasing hormone-stimulation test: a pharmacogenomic study. Thyroid 20: 1407-1412.

15. Asakura $Y$, Muroya K, Adachi M, Narumi S, Hasegawa $T$ (2010) TSH, free T3, and free T4 response to TRH stimulating test. Hormone to Rinsho 58: 47-52 (In Japanese).

16. Hattori N, Ishihara T, Ikekubo K, Moridera K, Hino M, et al. (1992) Autoantibody to human prolactin in patients with idiopathic hyperprolactinemia. J Clin Endocrinol Metab 75: 1226-1229.

17. Hadlow NC, Rothacker KM, Wardrop R, Brown SJ, Lim EM, et al. (2013) The relationship between TSH and free T4 in a large population is complex and nonlinear and differs by age and sex. J Clin Endocrinol Metab 98: 29362943.

18. Ueda Y, Tagami T, Tamanaha T, Kakita M, Tanase-Nakao $\mathrm{K}$, et al. (2015) A family of RTHbeta with p.R316C mutation presenting occasional syndrome of inappropriate secretion of TSH. Endocr J 62: 251-260.

19. Ohba K, Iwahara K, Unno $T$, Satoh $T$, Nakamura $H$ (2012) A patient with macro-thyrotropin with delayed thyrotropin and blunted free triiodothyronine response after thyrotropin-releasing hormone administration: a case report. Program of 82nd Scientific Sessions of American
Thyroid Association, A55 (Abstract).

20. Halsall DJ, Fahie-Wilson MN, Hall SK, Barker P, Anderson J, et al. (2006) Macro thyrotropin-IgG complex causes factitious increases in thyroid-stimulating hormone screening tests in a neonate and mother. Clin Chem 52: 1968-1969.

21. Mendoza H, Connacher A, Srivastava R (2009) Unexplained high thyroid stimulating hormone: a "BIG" problem. BMJ Case Rep 2009.

22. Hattori N, Ishihara T, Yamagami K, Shimatsu A (2015) Macro TSH in patients with subclinical hypothyroidism. Clin Endocrinol (Oxf) 83: 923-930.

23. Hattori N, Ishihara T, Shimatsu A (2016) Variability in the detection of macro TSH in different immunoassay systems. Eur J Endocrinol 174: 9-15.

24. Hattori N, Aisaka K, Chihara K, Shimatsu A (2018) Current thyrotropin immunoassays recognize macrothyrotropin leading to hyperthyrotropinemia in females of reproductive age. Thyroid 28: 1252-1260.

25. Kourides IA, Weintraub BD, Maloof F (1978) Large molecular weight TSH-beta: the sole immunoactive form of TSH-beta in certain human sera. J Clin Endocrinol Metab 47: 24-33.

26. Kadoya M, Koyama S, Morimoto A, Miyoshi A, Kakutani M, et al. (2017) Serum macro TSH level is associated with sleep quality in patients with cardiovascular risksHSCAA Study. Sci Rep 7: 44387.

27. Leow MK (2016) A review of the phenomenon of hysteresis in the hypothalamus-pituitary-thyroid axis. Front Endocrinol (Lausanne) 7: 64.

28. Beck-Peccoz P, Persani L, Mannavola D, Campi I (2009) Pituitary tumours: TSH-secreting adenomas. Best Pract Res Clin Endocrinol Metab 23: 597-606. 